\title{
EnEx-RANGE - Robust autonomous Acoustic Navigation in Glacial icE
}

\author{
Dirk Heinen ${ }^{1, \star}$, Dmitry Eliseev ${ }^{1}$, Christoph Henke ${ }^{2}$, Sabina Jeschke ${ }^{2}$, Peter Linder $^{1}$, Sebastian \\ Reuter $^{2}$, Sebastian Schönitz ${ }^{2}$, Franziska Scholz ${ }^{1}$, Lars Steffen Weinstock ${ }^{1}$, Stefan Wickmann ${ }^{1}$, \\ Christopher Wiebusch ${ }^{1}$, and Simon Zierke ${ }^{1}$ \\ ${ }^{1}$ III. Physikalisches Institut, RWTH Aachen University, Germany \\ ${ }^{2}$ IMA/ZLW \& IfU, RWTH Aachen University, Germany
}

\begin{abstract}
Within the Enceladus Explorer Initiative of the DLR Space Administration navigation technologies for a future space mission are in development. Those technologies are the basis for the search for extraterrestrial life on the Saturn moon Enceladus. An autonomous melting probe, the EnEx probe, aims to extract a liquid sample from a water reservoir below the icy crust.

A first EnEx probe was developed and demonstrated in a terrestrial scenario at the Bloodfalls, Taylor Glacier, Antarctica in November 2014. To enable navigation in glacier ice two acoustic systems were integrated into the probe in addition to conventional navigation technologies. The first acoustic system determines the position of the probe during the run based on propagation times of acoustic signals from emitters at reference positions at the glacier surface to receivers in the probe. The second system provides information about the forefield of the probe. It is based on sonographic principles with phased array technology integrated in the probe's melting head. Information about obstacles or sampling regions in the probe's forefield can be acquired. The development of both systems is now continued in the project EnEx-RANGE. The emitters of the localization system are replaced by a network of intelligent acoustic enabled melting probes. These localize each other by means of acoustic signals and create the reference system for the EnEx probe.

This presentation includes the discussion of the intelligent acoustic network, the acoustic navigation systems of the EnEx probe and results of terrestrial tests.
\end{abstract}

\section{Enceladus Explorer - EnEx}

The measurements by the CASSINI mission to the Saturn moon Enceladus discovered a global ocean below the icy crust [1]. Hence, it is a possible candidate for extraterrestrial life within our solar system. The mission identified active cryovolcanoes at Enceladus' south pole region. This region is a point of interest for a future mission to Enceladus.

The Enceladus Explorer Initiative (EnEx) managed by the DLR Space Administration aims for an Enceladus mission. Within this initiative navigation base technologies are developed. The idea is to develop a probe, which is able to navigate through ice, find a liquid water reservoir, take a sample and

^e-mail: heinen@physik.rwth-aachen.de 
analyze it. Several projects within the initiative focus on different navigation techniques.

A first EnEx probe for a terrestrial test scenario was developed in the years 2012 - 2015 and successfully demonstrated in 2014 [2]. For the demonstration a clean sample was retrieved from a water filled crack at a depth in about $20 \mathrm{~m}$ at the Blood Falls, Taylor Glacier, Antarctica.

For this scenario the carrier probe, the EnEx-IceMole, was equipped with navigation, decontamination and sampling systems. The EnEx-IceMole is able to drive curved trajectories through ice. To determine the position and orientation of the probe an acoustic positioning system, an inertial measurement unit and a magnetometer were integrated into the EnEx probe. To identify objects, e.g. stones or liquid water, in front of the probe the forefield was scanned with ultrasonic phased arrays. The EnEx probe was tested in glaciers in the Alps and Antarctica in advance of the final demonstration at the Blood Falls.

\section{EnEx-RANGE}

The EnEx-RANGE project is an interdisciplinary project between physics and engineering within the EnEx Initiative. The abbreviation RANGE stands for Robust autonomous Acoustic Navigation in Glacial icE.

The main subject of the project is the development of an autonomous acoustic sensor network (figure 1). The nodes of the network are Autonomous Pinger Units (APUs), which are described in section 3. In addition to the network the acoustic navigation systems for the forefield exploration and the positioning of the first EnEx probe will be enhanced. The new systems are going to be validated and demonstrated in collaboration with the other EnEx Initative projects in the Alps in 2017 and 2018.
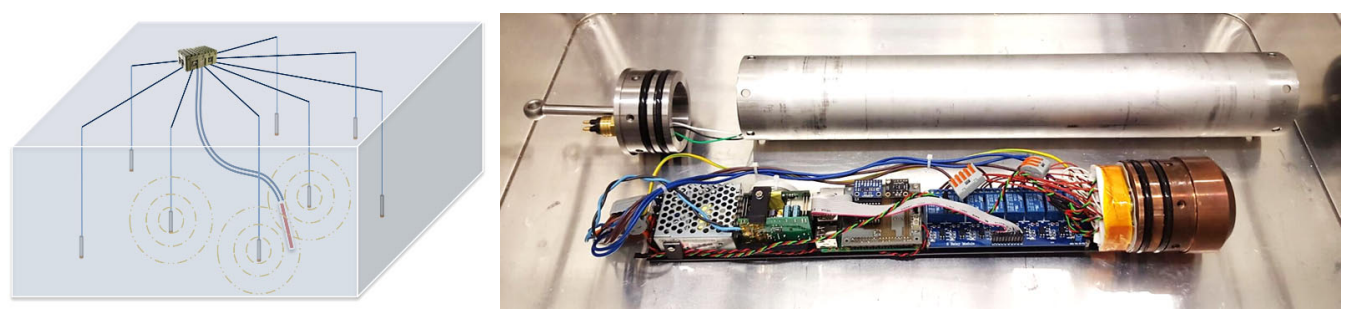

Figure 1. The scheme on the left side shows an artistic impression of the APU network (typically 50 to $100 \mathrm{~m}$ distance between APUs). The EnEx probe navigates through the network and determines its position in reference to the network. The picture on the right shows the first APU without acoustic instrumentation. This APU was successfully tested in the Alps in 2015 and 2016.

\section{APU Network}

The Autonomous Pinger Units (APU) are melting probes, equipped with local intelligence, an acoustic pinger, acoustic receivers, house keeping sensors and a melting control system. The APUs are able to communicate via acoustic signals and determine the distances between each APU by the propagation of these signals. This information is exchanged by the APUs to determine the relative position of each APU within the network. The local intelligence optimizes the APU network in size and positioning error. After the APU network is set up, it provides the reference for the EnEx probe to navigate trough.

The APU carrier system is mainly a watertight, pressure housing with an outer diameter of $8 \mathrm{~cm}$ and a length about $60 \mathrm{~cm}$ (figure 11). The melting head with heating elements allows the APU to 
melt vertically into ice at a speed of about $3 \mathrm{~m} / \mathrm{h}$. A stainless steel tube connects the melting head and the back plate. A cable, for power and communication, and a rope, to extract the probe after the scenario, are attached to the back plate. The APUs' control system is system on a chip (SoC) based. The SoC manages the heater control, the data acquisition, the signal generation and provides the computing power for the autonomy. For house keeping a MARG ${ }^{1}$ sensor, a pressure sensor and several temperature sensors are integrated into the carrier system. A first APU without the acoustic instrumentation was tested in test campaigns in summer 2015 and 2016 on glaciers in the Alps.

The acoustic pinger design aims for long-range communications between the APUs. To achieve such a long range the pinger will be optimized to a high output power with respect to the limitation in size within the APU carrier system. For a good coupling between the pinger and ice, the acoustic transducer is pressed against the melting head. The acoustic transducer consists of a stack of lead zirconate titanate (PZT) rings. The APUs' SoC controls an arbitrary function generator for signal generation. These signals are amplified by a transistor based, high power amplifier and fed to the transducers. A transducer integrated into an APU was first tested in the 2016 test campaign.

The design of the acoustic receivers is matched to the frequency range of the pingers' signal frequency. Three acoustic transducers, lead zirconate titanate (PZT) disks, are glued on the inner sides of the APU tube. Each transducer is coupled to front end electronics, which filter, amplify and digitize the signals. For first in ice measurements an APU instrumented with the acoustic receivers was tested in the 2016 test campaign. To test the frequency response of the APU receivers coupled to glacial ice, signals were emitted by a reference transducer and received by a demonstrator APU over a short distance of about $1 \mathrm{~m}$. Furthermore the communication between the APU pinger and the APU receivers was successfully demonstrated (figure 2).

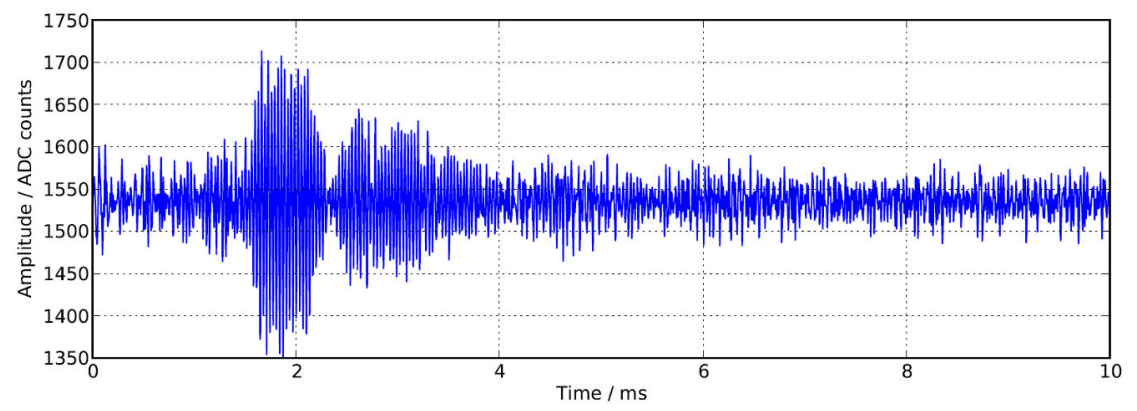

Figure 2. The plot shows a waveform measured during a test campaign on the Langenferner glacier in the Alps in 2016. The acoustic signal was emitted by an APUs' pinger and measured by another APUs' receiver.

The network is designed to be able to configure itself autonomously and to compensate system failures of individual APUs. Each single APU is controlled by a software agent. This agent is realized by a rule-based expert system. The expert system evaluates available information of the environment and creates an internal world model. This internal world model is shared with the other APUs. Based on the shared world model each APU decides its further actions. The APU positions are iteratively determined to define the network topology. To study different scenarios depending on various constraints (e.g. fixed starting positions of APUs, air filled cracks, inhomogeneities in ice quality, size of APU network) simulation modules for all APU components and a physical model of the ice are

\footnotetext{
${ }^{1}$ MARG, acronym for Magnetic, Angular Rate, and Gravity
} 
available. The first test of an APU network in ice will be in 2017 in the Alps. For this test in total seven fully instrumented APUs will be available and form the network.

\section{Acoustic Positioning}

Similar to the APU, an APU SoC and four acoustic receivers will be integrated into the EnEx probe. The APUs will be able to share their internal world model with the information about their positions. By recording and analyzing the acoustic signals from each APU, the EnEx probe will determine its position in reference to the network.

\section{Acoustic Forefield Exploration}

This system provides information about the forefield of the EnEx probe. It is based on sonographic principles with transducer arrays integrated in the probe's melting head. By modulation of the phase of the acoustic signal generation at each transducer the acoustic beam can be steered and focused. Any objects, e.g. stones, crevasses or liquid water, in front of the probe reflect this signal. The reflections are acquired at the transducers and visualized for the operator in a B-scan. After the identification of an object, the operator is able to act, e.g. steer the probe around a stone.

\section{Conclusion and Outlook}

The APU network is a robust approach for navigation within a glacier. The APUs' hardware is in development and a first network with seven APUs will be demonstrated in a test campaign in 2017. The EnEx probe, with the acoustic navigation systems, was successfully demonstrated in a terrestrial scenario and will take part in the up coming test campaigns.

Furthermore, synergies exist between the EnEx activities and research projects within the ARENA community. At the moment we plan to support the ARIANNA project [4] with the development of an APU like probe for deploying antennas at a depth of about $100 \mathrm{~m}$ into the Ross Ice Shelf. Furthermore, we developed an acoustic sensor based on the EnEx receiver concept for the IceCube-Gen2 neutrino detector for module positioning and acoustic neutrino detection [5].

\section{Acknowledgment}

The Enceladus Explorer Initiative is managed by the DLR Space Administration. The EnEx-RANGE project is funded by the German Federal Ministry of Economics and Energy (BMWi) by resolution of the German Federal Parliament under the funding code 50NA1501.

\section{References}

[1] D. A. Patthoff and S. A. Kattenhorn, Geophys. Res. Lett. 38, L18201 (2011)

[2] J. Kowalski et al., Cold Regions Science and Technology 123, 53-70 (2016)

[3] D. Eliseev et al., Annals of Glaciology 55(68), 253-259 (2014)

[4] A. Nelles et al., EPJ WoC, in these proceedings, ARENA 2016

[5] S. Wickmann et al., EPJ WoC, in these proceedings, ARENA 2016 